\title{
Zur Industriegeschichte Rottweils und im Raum Schwarzwald-Baar-Heuberg
}

\author{
Einführung*
}

\author{
Von Gert Kollmer-von Oheimb-Loup
}

Wenn man über Wirtschaftsregionen im Südwesten spricht, so denkt man zunächst an die Großregion Stuttgart oder an den Rhein-Neckar-Raum. Unbestritten stand der Mittlere Neckarraum in der Industriegeschichte des Landes Baden-Württemberg an erster Stelle und ist es bis heute geblieben ${ }^{1}$. Weniger denken wir an die Region Schwarzwald-Baar-Heuberg im Regierungsbezirk Freiburg. Sie besteht aus den drei seit der Kreisreform von 1973 festgelegten Landkreisen SchwarzwaldBaar-Kreis, Rottweil und Tuttlingen². Dieses Gebiet, das sich heute „Gewinnerregion“ nennt, zeichnet sich seit mehreren Jahrzehnten durch eine hohe, deutlich über dem Landesdurchschnitt liegende, mittelständisch geprägte Industriedichte ${ }^{3}$ aus $^{4}$. Es erstreckt sich mit insgesamt 76 Städten und Gemeinden, Villingen-Schwenningen als Oberzentrum sowie den Städten Donaueschingen, Rottweil, Schramberg und Tuttlingen als Mittelzentren vom Hochschwarzwald im Westen bis zum Bereich des Großen Heubergs auf der Schwäbischen Alb im Osten, im Süden grenzt es an den Schweizer Kanton Schaffhausen.

\footnotetext{
* Der Beitrag basiert auf der Einführung in die Arbeitsgruppe „Zur Industriegeschichte Rottweils und im Raum Schwarzwald-Baar-Heuberg", die im Rahmen der 62.Jahrestagung der Kommission für geschichtliche Landeskunde in Baden-Württemberg am 26. Juni 2015 in Rottweil gestaltet wurde.

1 Baden-Württemberg 2000. Der neue Atlas für das ganze Land, hg. vom Statistischen Landesamt Baden-Württemberg, Stuttgart 1999, S.118ff.

${ }^{2}$ Karl Heim/Wolf-Rüdiger Michel/Guido Wolf, Kräfte bündeln, Grenzen überwinden, in: Schwarzwald-Baar-Heuberg. Drei Landkreise - Eine starke Wirtschaftsregion (Monographien deutscher Wirtschaftsgebiete), Oldenburg 2003, S.12 ff.

${ }^{3}$ Industriedichte gibt die Anzahl der Beschäftigten je 1.000 Einwohner an.

${ }^{4}$ Vgl. dazu Heinz Rudi Link/Anton Villing, Hidden Champions in der Gewinnerregion, in: Schwarzwald-Baar-Heuberg (wie Anm.2) S. $58 \mathrm{ff}$.
} 
Gewiss erreichte die allgemeine Industrialisierung erst spät die Region. Die Industrieunternehmen entwickelten sich überwiegend aus kleingewerblichen Strukturen, weshalb hier der Aufschwung, sieht man von einigen wenigen Branchen wie Uhren, Pulver oder Musikinstrumente ab, erst ab Ende des 19. Jahrhunderts stattfand ${ }^{5}$. Bei Betrachtung der kleinstädtischen Großunternehmen Württembergs wird jedoch deutlich, dass in den 1930er Jahren von 29 Unternehmen mit über 1.000 Beschäftigten sieben, das entspricht beinahe einem Viertel, hier beheimatet waren. Davon standen die Uhrenfabrik Junghans mit ca. 8.500 Beschäftigten an erster, die Waffenfabrik Mauser mit über 7.100 Beschäftigten an zweiter und die Harmonikafabrik Hohner mit 4.100 Mitarbeitern an siebter Stelle. Die Tuttlinger Firmen wie das medizintechnische Unternehmen Jetter \& Scherer und die Schuhfabrik Riecker sowie die Rottweiler Pulverfabrik mit zwischen 1.500 und 1.800 Mitarbeitern belegten die Plätze 13, 14 und $17^{6}$.

Was diese Wirtschaftsregion in ihrer Industriegeschichte gegenüber anderen in Deutschland mit am stärksten auszeichnet, ist die erfolgreiche Überwindung mehrerer Strukturwandel in den letzten einhundert Jahren. Die wichtigsten Industriestandorte wie Rottweil, Schramberg, Oberndorf, Tuttlingen, Trossingen und Schwenningen mit ihrem Umfeld waren vor dem Ersten Weltkrieg industriell geprägt außer von den bereits genannten Großbetrieben u.a. von Uhrenfabriken wie Kienzle und Mauthe. Sehr schnell bildeten sich Cluster und es kamen kleinere Zulieferer und aus dem Handwerk entstandene Industriebetriebe der Uhrenfertigung, dem Maschinenbau und der Metallwarenherstellung hinzu. Aus Mauser und seiner Waffenfabrik heraus entstanden etliche Firmen, die auf ihrem Gebiet der Metallverarbeitung, der Feinmechanik und der Präzisionstechnik erfolgreich wurden und heute europaweit operieren, teilweise sogar Weltmarktführer sind ${ }^{7}$. Sieht man von der Pulverfabrik ab, waren die meisten Industriezweige von der Feinmechanik bestimmt.

${ }^{5}$ Arik Piening, Schwarzwälder Uhrenindustrie zwischen Aufschwung und Niedergang. Regionalgeschichtliche Analyse der hausgewerblichen und industriellen Phase über drei Jahrhunderte, masch. Magisterarbeit Universität Hohenheim 2012, S. 43 ff. Helmut KAHLERT, 300 Jahre Schwarzwälder Uhrenindustrie, Gernsbach 1986, S. 169 ff. Ausführlich dazu: Julius KUсKUсK, Die Uhrenindustrie des Württembergischen Schwarzwaldes, Tübingen 1906, S.49-73. Ferner Paul Dienstag, Die deutsche Uhrenindustrie. Eine Darstellung der technischen Entwicklung in ihrer volkswirtschaftlichen Bedeutung (Technisch-volkswirtschaftliche Monographien, Bd.11), Leipzig 1910, S.40-105 und S.141-151. Hartmut Berghoff, Zwischen Kleinstadt und Weltmarkt. Hohner und die Harmonika 1857-1961. Unternehmensgeschichte als Gesellschaftsgeschichte, Paderborn u.a. 1997, S.68ff. Dazu auch: Baden-Württemberg - Das Land in seinen Kreisen. Der Landkreis Rottweil, Bd.1, Ostfildern 2003, S.195. Willi A. Boelcke, Landschaft und Bevölkerung im Spiegel der Wirtschaftsgeschichte, in: Wirtschaftsraum Schwarzwald-Baar-Heuberg (Monographien deutscher Wirtschaftsgebiete), Oldenburg 1989, S. $10 \mathrm{ff}$.

${ }^{6}$ Siehe dazu Berghoff, Zwischen Kleinstadt (wie Anm. 5) S. 28 f.

7 Baden-Württemberg - Das Land in seinen Kreisen. Der Landkreis Rottweil (wie Anm. 5) S. 62. 
Der Wirtschaftsraum Schwarzwald-Baar-Heuberg hatte schon nach dem Zweiten Weltkrieg im Südwesten einen vergleichsweise hohen Beschäftigungsbesatz und weist in jüngster Zeit nach dem Ballungsraum Stuttgart die zweithöchste Industriedichte in Baden-Württemberg auf ${ }^{8}$. Um diese Stellung zu erhalten, musste sich die Industrie einem enormen Strukturwandel unterziehen. Viele traditionsreiche Betriebe hatten ihre einstmalige Bedeutung verloren oder gar aufgehört zu existieren. Nachgerückt sind innovative Unternehmen, deren High-Tech-Produkte nach und nach die herausragende Stellung der Region bis zum heutigen Zeitpunkt prägen. Ein entscheidender Vorteil war, dass die heimische Wirtschaft stets auf hochqualifizierte Arbeitskräfte zurückgreifen konnte. Die jüngste Entwicklung zeigt sehr eindrucksvoll, dass aus der tradierten Branchenstruktur heraus, meist mit fließenden Übergängen, eine ausgesprochene HighTech-Orientierung gelungen ist ${ }^{9}$.

Es begann alles nach dem Ersten Weltkrieg. Auch diese Region unterlag der Krisenanfälligkeit der Wirtschaft der 1920er Jahre, die zum Großteil in der Industriestruktur begründet war. Zum einen erfolgte eine starke Konzentration auf wenige Industriezweige und zum anderen unterlagen gerade diese starken Absatzschwankungen, wie z.B. die Uhren- und Rüstungsindustrie. Die latent schwierige Konjunkturlage der Weimarer Zeit und die Weltwirtschaftskrise ließen dies in besonderer Weise offenbar werden. Hinzu kam, dass der Versailler Vertrag die Rüstungsindustrie zwang, auf Friedensprodukte umzustellen, was wiederum zur Hinwendung an neue Produkte führte, wie z. B. bei Mauser zur Produktion von Automobilen, Näh- und Rechenmaschinen sowie Meßwerkzeugen ${ }^{10}$ oder wie bei der Rottweiler Pulverfabrik zur Herstellung von Kunstseide ${ }^{11}$. Während die Uhrenindustrie nach dem Zweiten Weltkrieg zuletzt durch die Revolution der Quarztechnik nahezu bedeutungslos wurde, veränderte sich hier die Wirtschaftsstruktur innerhalb weniger Jahre. Aus einer Branchenkrise wurde eine Regionalkrise.

Die Erholung setzte jedoch relativ schnell ein. War Anfang der 1960er Jahre die Uhrenindustrie gemessen an der Beschäftigtenzahl noch die führende Branche, rückten vor allem die elektrotechnische Industrie sowie der Maschinen- und Fahrzeugbau, die Feinmechanik und Optik auf die ersten Plätze ${ }^{12}$. Aus den Entwick-

${ }^{8}$ Rudolf Kunbach, Wirtschaftsraum mit Zukunft - das Branchenspektrum in der Region Schwarzwald-Baar-Heuberg, in: Schwarzwald-Baar-Heuberg (wie Anm.2) S. 96.

9 Baden-Württemberg - Das Land in seinen Kreisen. Der Landkreis Rottweil (wie Anm. 5) S. 197.

10 Ebd., S. 62.

11 Hans Ulrich Lutz, Die 50 Rhodia-Jahre im Rottweiler Neckartal, in: Industriekultur im Neckartal Rottweil. Vom Pulver über Nylon zur gewerblichen Vielfalt, hg. von Stefan KING/Hermann KLos, Rottweil 2012, S. 12 ff.

12 Landesentwicklungsbericht Baden-Württemberg 1994, Stuttgart 1995, S.141. Franz Mussler, Bevölkerung und Wirtschaft, in: Der Kreis Rottweil, Aalen 1963, S. 201 ff. BadenWürttemberg - Das Land in Kreisen. Der Landkreis Rottweil (wie Anm. 5) S. 195 f. 
lungen in der Uhrenindustrie entstand mit der Elektro- und Magnettechnik ein eigenständiger Industriezweig, dessen Produkte eine ständig steigende Nachfrage erfuhren. Die Elektrotechnik- und Computerbranche hat in der Region ihre Wurzeln im Bereich der traditionellen Zeiterfassungsgeräte. So entstanden Unternehmen der Kommunikationstechnik, andere entwickelten und produzierten technisch hochwertige Zeitmesser, Zählwerke, Drehgeber oder Zähler für Positionierungssysteme in der Automatisierungsindustrie. Stets war es die Präzisionstechnik wie die weltberühmt gewordene Tuttlinger Medizintechnik oder die Phonoindustrie, die es mit Plattenspielern, Radio-, Tonband- und Fernsehgeräten, Lautsprechern, Videorekordern oder CD Spielern schaffte, verlorengegangene Märkte durch neue zu ersetzen. Der Strukturwandel setzte sich weiter fort. Viele Unternehmen aus diesem Wirtschaftsgebiet verstanden es, sich aus ihren Produktionsfeldern heraus innovativ weiter zu entwickeln und damit zum Siegeszug der Hochtechnologie zu verhelfen, wie Produkte im Bereich der Lasermessung, Programmier- und Steuerungsverfahren oder Robotersysteme zeigen ${ }^{13}$.

Die beiden folgenden Beiträge beschäftigen sich im Besonderen mit der Pulverindustrie, die über viele Jahrzehnte für die Wirtschaft dieser Region stand und eine hohe volkswirtschaftliche Relevanz besaß. Sie ist signifikant für den vorher beschriebenen gewaltigen Strukturwandel, den diese Wirtschaftsregion im 20. Jahrhundert erfuhr. Die Pulverindustrie, die der württembergische Industriepionier Max Duttenhofer in Rottweil etablierte, ist heute verschwunden, wie etwa auch die Harmonikaindustrie nahezu bedeutungslos geworden is $\mathrm{t}^{14}$.

${ }^{13}$ Ralf Ketterer, Vom Orchestrion zum Fernsehgerät. Zur Geschichte der Herstellung von Unterhaltungsapparaten in Villingen seit 1865, in: Schwarzwälder Wertarbeit - Beiträge zur Wirtschaftsgeschichte in Villingen und Schwenningen. Zwischen Kopfhörer und Trachtenhaube, Bd. 1 (Veröffentlichungen des Stadtarchivs und der Städtischen Museen VillingenSchwenningen, Bd.25,1), Villingen-Schwenningen 2002, S. 13 ff. Ferner ebd., S. 64 ff. Kurt Kositzke/Ulrich HäsLER, Vielfältige Industrie, in: Wirtschaft im Südwesten. Zeitschrift der Industrie- und Handelskammer Hochrhein-Bodensee, Schwarzwald-Baar-Heuberg, Südlicher Oberrhein 7 (1997) S. $10 \mathrm{ff}$.

${ }^{14}$ Der auf der Kommissionstagung in Rottweil gehaltene Vortrag von Martin Häffner über „Hohner und die Harmonikaindustrie in Südwestdeutschland“ konnte hier leider nicht zum Abdruck kommen. 